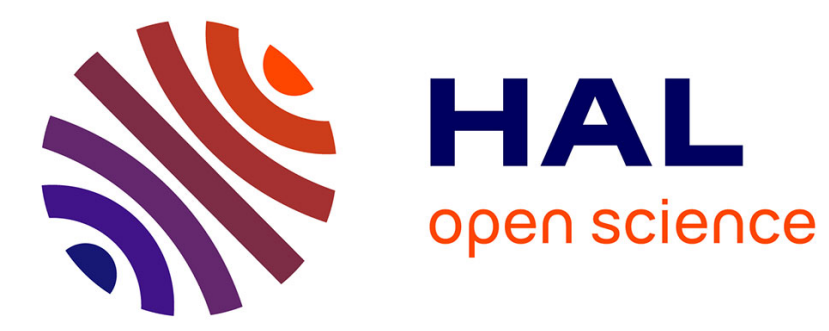

\title{
Les fièvres hémorragiques virales
}

Pierre Tattevin, Gisèle Lagathu, Matthieu Revest, Christian Michelet

\section{To cite this version:}

Pierre Tattevin, Gisèle Lagathu, Matthieu Revest, Christian Michelet. Les fièvres hémorragiques virales. Revue Francophone des Laboratoires, 2016, Infections ostéo-articulaires, 2016 (480), pp.7180. 10.1016/S1773-035X(16)30091-0 . hal-01299296

\section{HAL Id: hal-01299296 \\ https://hal-univ-rennes1.archives-ouvertes.fr/hal-01299296}

Submitted on 31 May 2016

HAL is a multi-disciplinary open access archive for the deposit and dissemination of scientific research documents, whether they are published or not. The documents may come from teaching and research institutions in France or abroad, or from public or private research centers.
L'archive ouverte pluridisciplinaire HAL, est destinée au dépôt et à la diffusion de documents scientifiques de niveau recherche, publiés ou non, émanant des établissements d'enseignement et de recherche français ou étrangers, des laboratoires publics ou privés. 


\section{Les fièvres hémorragiques virales}

\section{Titre anglais : Viral Haemorragic fever}

Pierre Tattevin, ${ }^{1}$ Gisèle Lagathu, ${ }^{2}$ Matthieu Revest, ${ }^{1}$ Christian Michelet ${ }^{1}$

${ }^{1}$ Maladies Infectieuses et Réanimation Médicale

CHU Pontchaillou, Rue Louis Guilloux, 35000 Rennes, France

${ }^{2}$ Virologie

CHU Pontchaillou, Rue Louis Guilloux, 35000 Rennes, France

Auteur correspondant : pierre.tattevin@ chu-rennes.fr 


\section{Résumé}

Les fièvres hémorragiques virales regroupent plusieurs viroses, potentiellement graves, avec un tableau clinique relativement stéréotypé, d'évolution biphasique. Après une incubation de 2 à 21 jours, apparition d'un syndrome pseudo-grippal qui évolue au bout de quelques jours, de manière imprévisible, chez une proportion variable de patients, vers un syndrome hémorragique avec choc. La transmission inter-humaine s'effectue le plus souvent par contacts avec des liquides biologiques, surtout en présence de sang. La biologie de ces virus, leur épidémiologie et leur létalité sont très variables. Nous détaillons dans cette revue i) deux filovirus - Ebola et Marburg - très proches (réservoirs primates non humains et chauves-souris, épidémies fulgurantes en Afrique sub-Saharienne, létalité $40-90 \%)$; ii) deux bunyavirus - le virus de la fièvre Crimée-Congo et celui de la fièvre de la vallée du Rift - arboviroses transmissibles par des tiques (Crimée-Congo) ou des moustiques (Vallée du Rift), avec un large réservoir de vertébrés; iii) un arénavirus - le virus de la fièvre de Lassa - avec comme réservoir des rongeurs en Afrique de l'Ouest, le seul virus de cette liste transmissible par voie respiratoire. Pour toutes ces fièvres hémorragiques virales, l'importance d'un diagnostic rapide réside plus dans la nécessité d'interrompre les chaines de transmission que dans les possibilités thérapeutiques, limitées aux traitements symptomatiques, à l'exception notable de la fièvre de Lassa (efficacité probable de la ribavirine).

Mots-clés: fièvre de Lassa; fièvre de la Vallée du Rift; fièvre de Marburg; Ebola; fièvre hémorragique Crimée-Congo 


\section{Abstract}

Viral haemorragic fevers are characterized by similar clinical manifestations, with a biphasic course : After a 2-21 day-incubation, onset of an influenza-like illness, that will drive ti an haemorragic syndrom with shock, in a subgroup of patients, unpredictable. Human-to-human transmission mostly occurs through contact with biological fluids, especially if contaminated with blood. The biology, the epridemiology and the letality of these viruses differ. We present in this review i) two filoviruses - Ebola and Marburg - very similar (non-human primates and bats as the main reservoir, dramatic outbreaks in sub-Saharian Africa, 40-90\% letality rates); ii) two bunyaviruses - Crimean-Congo haemorragic fever, and Rift valley fever viruses - arboviruses transmissible by ticks (Crimean-Congo haemorragic fever), or mosquitoes (Rift valley fever), with broad reservoirs of vertebrates; iii) one arenavirus - Lassa fever virus - with rodents from west Africa as the main reservoir, the only virus in this list with potential respiratory transmission. For all these viral haemorragic fevers, the importance of an early diagnosis relies mostly in the need to interrupt human-to-hulan transmission, as therapeutic options are mostly restricted to symptomatic treatment, with the exception of Lassa fever (ribavirin probably effective).

Keywords: Lassa fever; Rift valley fever; Marburg fever; Ebola; Crimean-Congo haemorragic fever 


\section{Généralités}

Les fièvres hémorragiques virales forment un groupe de maladies virales hétérogènes, regroupées de manière syndromique afin de faciliter la démarche diagnostique face à un cas suspect. Le tableau classique comporte, à des degrés divers, une fièvre, des signes hémorragiques et une défaillance circulatoire. Ces maladies se caractérisent en général par une évolution biphasique, avec une première phase de quelques jours dominée par un syndrome pseudo-grippal sans spécificité, puis une seconde phase souvent rapidement évolutive marquée par des signes hémorragiques diffus parfois impressionnants : hémorragies cutanées ou cutanéo-muqueuses rapidement extensives, suffusions hémorragiques en regard de points de ponction même anciens, et hémorragies viscérales souvent fatales (hématémèse, épistaxis, hémoptysies, rectorragies, etc.). Un état de choc multi-factoriel accompagne souvent ces phases hémorragiques. II est très difficile de distinguer les différentes fièvres hémorragiques virales d'après la présentation clinique, même si certaines particularités méritent d'être signalées (prédominance des signes digestifs avec Ebola, atteinte ophtalmologique au cours de la fièvre de la Vallée du Rift, rhabdomyolyse au cours de la fièvre Crimée-Congo). En général, la nature du virus responsable est soupçonnée d'après la provenance du patient, en fonction des zones d'endémies/d'épidémies, qui sont évolutives.

Les virus responsables de ces syndromes sont tous des virus à ARN simple brin, possédant une enveloppe lipidique, mais leurs morphologies sont très différentes et ils sont issus de familles bien distinctes (tableau I). Dans cette mise au point, après un bref rappel des principes de prise en charge d'une suspicion de fièvre hémorragique virale importée en France, nous détaillerons les caractéristiques virologiques, épidémiologiques et cliniques des viroses Ebola, Marburg, Lassa, Crimée-Congo et Fièvre de la vallée du Rift, qui sont celles qui présentent la plus forte probabilité d'être importées en France. Les Flavivirus (fièvre jaune, dengue hémorragique) ne seront pas abordés dans cette revue. 


\section{Prise en charge d'une suspicion de fièvre hémorragique virale importée en France}

Devant la sévérité des épidémies de fièvres Ebola ou Marburg en Afrique, les antécédents d'importation de ces pathogènes dans d'autres pays d'Europe ou d'Amérique du Nord, et la transmission nosocomiale parfois au premier plan lors des épidémies, le Conseil supérieur d'hygiène publique de France avait émis en 2002 un rapport intitulé Infections virales aiguës importées hautement contagieuses et leur prise en charge (http://editions.ensp.fr). Ce rapport a été rédigé alors qu'aucun cas de fièvre hémorragique virale n'avait été importé en France, mais il était prévu une révision de ce rapport en fonction de l'expérience acquise au fil des années, notamment lorsque cette situation serait rencontrée, sur le principe des retours d'expérience. Le diagnostic à Rennes, en 2004, d'une fièvre hémorragique Crimée-Congo importée, avait été une 'première' $(1,2)$, mais c'est avec l'épidémie 'Ebola' en Afrique de l'Ouest en 2014, que la France a mené une réflexion en profondeur sur les modalités d'accueil des patients suspects de fièvre hémorragique virale et leur prise en charge clinico-biologique.

Les grands principes qui ont guidé les différentes recommandations de prise en charge de ces cas suspects découlent des règles suivantes : i) la transmission inter-humaine des fièvres hémorragiques virales s'effectue par contact avec du sang ou des liquides biologiques contaminés dans l'immense majorité des cas (seule la fièvre de Lassa peut se transmettre par voie respiratoire, parmi celles qui sont détaillées ici); ii) la co-existence de signes hémorragiques et d'une virémie élevée rend ce risque majeur chez les patients les plus graves ; iii) les mesures de protection des soignants, certes indispensables, ne doivent pas représenter une perte de chance pour les patients suspects de fièvre hémorragique virale. Ce dernier point est d'autant plus important que ces patients 'suspects' sont, le plus souvent, atteints d'une autre pathologie infectieuse pour laquelle le retard de prise en charge peut engager le pronostic (accès palustre, sepsis grave, etc.).

Le risque d'importation des virus responsables de fièvre hémorragique est différent selon le type de virus, le temps d'incubation et la rapidité d'installation des signes hémorragiques. Il faut savoir évoquer une fièvre hémorragique virale devant tout patient présentant une fièvre et des signes hémorragiques dans les trois semaines qui suivent un séjour à l'étranger dans un pays 'à risque'. Malheureusement, la multiplicité des zones géographiques dans lesquelles des cas de fièvre hémorragique ont été rapportés ces dernières années et l'accélération du rythme des alertes liées aux maladies infectieuses émergentes 
ne permet pas de proposer une liste restrictive des zones à risque : celle-ci serait rapidement périmée. Par contre, en cas de suspicion, le contact téléphonique direct et immédiat avec le centre national de référence $(\mathrm{CNR})$ des fièvres hémorragiques virales est hautement recommandé, car il permet de confronter le tableau clinique et la provenance du patient aux données récentes concernant la répartition des fièvres hémorragiques virales. De plus, si le doute persiste, ce contact permet d'organiser un acheminement et une analyse rapide des prélèvements. Les coordonnées du CNR figurent sur le site : http://www.pasteur.fr/fr/recherche/virologie/unites-et-groupes/biologie-infections-virales-emergentes

\section{La maladie virale Ebola}

Les virus Ebola et Marburg appartiennent à la famille des Filoviridae, genre Filovirus et sont parmi les pathogènes les plus virulents pour l'homme et les grands singes. Leur aspect en microscopie électronique est très caractéristique avec un aspect de longs filaments (Figure 1). II s'agit de virus enveloppés, fragiles avec une persistance dans l'environnement pouvant aller de quelques heures à quelques jours sur support solide. La concentration virale dans les fluides biologiques est très élevée : $10^{7}$ unités $/ \mathrm{ml}$ de sang. Le virus Ebola compte 5 espèces distinctes illustrant la grande diversité de ce virus: i) Ebolavirus Bundibugyo (BEBOV), Ebola Zaïre (ZEBOV), Ebolavirus Reston (REBOV), Ebolavirus Soudan (SEBOV), Ebolavirus Taï Forest (CIEBOV). Seules les espèces Zaïre et Soudan sont responsables d'importantes flambées épidémiques en Afrique.

Le virus Ebola aura été sans conteste le pathogène vedette des années 2014-2015, avec plus de 28000 cas cette année-là, dont plus de 11000 sont décédés (létalité estimée à $40 \%$ ) selon les chiffres officiels de l'OMS, très probablement sous-estimés (3). Le personnel soignant a payé un lourd tribut lors de cette épidémie avec 881 cas dont 531 décès (létalité à 60\%). Les premières épidémies de fièvres Ebola ont été rapportées lors de 2 flambées épidémiques successives en République Démocratique du Congo (au bord de la rivière 'Ebola', qui traverse la ville de Yambuku) en 1976, puis au Soudan, et en Ouganda en 1977. ,Par la suite, de multiples épidémies ponctuelles, sans facteur déclenchant évident, ont été observées, le plus souvent dans des zones rurales d’Afrique Centrale (figure 2). Les caractéristiques de ces épidémies étaient relativement superposables, avec une fièvre quasi-constante $\left(>38.3^{\circ} \mathrm{C}\right.$ dans plus de $99 \%$ des cas), une évolution biphasique (syndrome pseudo- 
grippal initial, évoluant brutalement vers une forme hémorragique au bout de quelques jours chez une partie des patients, sans qu'on puisse réellement prédire lesquels), et une létalité comprise entre 50 et $70 \%$. Les modalités d'acquisition du virus par le cas index de ces épidémies ne sont jamais parfaitement élucidées a posteriori, mais font très probablement intervenir un contact avec un des réservoirs (primates non humains et chauves-souris frugivores). Au décours, les catalyseurs de ces épidémies reposent sur une transmission inter-humaine par contact direct via les soins prodigués aux patients en phase hémorragique (transmission aux soignants et à l'entourage), ainsi que les pratiques funéraires non protégées $(4,5)$. II n'existe aucune donnée scientifique suggérant la possibilité d'une transmission par voie respiratoire, mais tous les liquides biologiques doivent être considérés comme contaminants en phase hémorragique, compte tenu de la forte virémie documentée à ce stade, qui se traduit par la possibilité d'isoler du virus dans tous les liquides potentiellement contaminés par du sang, même en faible quantité. La transmission inter-humaine ne peut avoir lieu qu'à partir de patients symptomatiques, à l'exception notable de la transmission sexuelle. En effet, le virus Ebola peut être isolé dans les secrétions génitales des patients convalescents pendant au moins 3 mois, avec des cas documentés de transmission sexuelle très probable, à distance de la maladie.

Une fois reconnues, et au prix d'interventions parfois musclées d'organisations internationales ou nationales, toutes les épidémies des années 1976-2013 ont pu être interrompues au bout de quelques semaines, essentiellement par la mise en place de mesures de prévention de la transmission interhumaine pré- et post-mortem, en l'absence de traitement préventif ou curatif (6,7). De 1976 à 2013, on estime qu'environ 1500 personnes au total sont décédées de la maladie Ebola, l'épidémie la plus importante ayant affecté 425 patients.

Lorsque les équipes de Médecins Sans Frontière, en collaboration avec le gouvernement Guinéen et l'OMS, ont signalé en mars 2014 l'existence d'une épidémie d'Ebola en Guinée-Konakry, il n'y avait pas de raison majeure de penser que cette épidémie allait se démarquer aussi nettement des précédentes (8). De fait, les courbes d'incidence des cas d'Ebola ont initialement suivi l'évolution classique, avec une nette tendance à la diminution à partir de mi-avril. Cependant, en quelques semaines, l'épidémie a redoublé d'intensité et s'est alors rapidement étendue à l'ensemble du territoire Guinéen et à 2 pays voisins (Sierra Leone et Libéria), jusqu'à être déclarée 'hors de contrôle' par MSF, 
motivant une alerte de l'OMS en août 2014, suivie d'une mobilisation majeure de l'aide internationale, financière, matérielle et humaine, reposant principalement sur le tri et l'isolement précoce des patients dans des centres dédiés et la sécurisation des enterrements, permettant un fléchissement très spectaculaire de la transmission inter-humaine, même si une transmission active a persisté, à bas bruit, dans ces 3 pays, avec quelques nouveaux cas diagnostiqués chaque semaine jusqu'à l'été 2015. En France, plus de 250 cas suspects d'Ebola ont été signalés entre le printemps 2014 et le printemps 2015 , en général sur la notion d'une fièvre $>38^{\circ} \mathrm{C}$ apparue moins de 21 jours après un séjour en zone d'épidémie (essentiellement, Libéria, Guinée et Sierra Leone). Aucun diagnostic d'Ebola n'a été posé en France au cours de cette épidémie, mais 2 patients présentant une maladie Ebola documentée en Guinée et en Sierra Leone ont été rapatriés à l'Hôpital Bégin, où ils ont tous les 2 guéris, sans transmission secondaire. D'autres pays industrialisés ont connu moins de succès, avec des décès et des transmissions autochtones, notamment l'Espagne et les Etats-Unis. Ces évolutions défavorables dans des pays disposant de moyens comparables à la France illustrent bien la virulence et les capacités de transmission de ce virus, et impliquent que la vigilance reste de mise. Compte-tenu de la rapidité d'évolution des informations et des procédures dans ce domaine, nous ne les détaillons pas dans ce chapitre, en invitant le lecteur à consulter le site de l'Institut de Veille Sanitaire (http://www.invs.sante.fr) et celui de la Société de Pathologie Infectieuse de Langue Française (SPILF, http://www.infectiologie.com), notamment l'onglet 'COREB'. Les critères diagnostiques (9), et les traitements (10), ont fait l'objet de mises au point. Pour les cliniciens amenés à prendre en charge ces cas suspects ou avérés, les principaux messages sont : i) la suspicion d'Ebola ne doit pas représenter une perte de chance pour les patients (les diagnostics de paludisme ou de sepsis grave d'origine bactérienne, nettement plus fréquents, doivent être systématiquement évoqués et traités empiriquement le cas échéant) ; ii) le traitement symptomatique et la compensation des défaillances sont indispensables et nécessitent souvent une réanimation intensive (cf. $>10 \mathrm{~L}$ de pertes digestives quotidiennes dans certaines observations), malgré la lourdeur des procédures visant à assurer l'absence de transmission inter-humaine et la protection des soignants.

\section{La Fièvre de Marburg}


Les virus responsables de la maladie virale Ebola et de la fièvre de Marburg étant très proches, les caractéristiques de ces 2 maladies sont similaires en termes de modalités de transmission, de dynamique des épidémies, de présentation clinique, de pronostic et de traitement. Le virus responsable de la fièvre de Marburg a été découvert à l'occasion d'une épidémie survenue en Europe en 1967 au sein d'équipes de chercheurs qui travaillaient sur des prélèvements biologiques de singes verts africains (Cercopithecus aethiops) importés d'Ouganda (11). Cette première épidémie a été responsable de 7 décès (sur 31 cas), en Allemagne (Marburg, Francfort) et en Serbie (Belgrade). Outre les cas acquis par contact direct avec les prélèvements contaminés, 6 cas secondaires ont été rapportés (soignants, ou partenaires des cas index), ce qui illustrait déjà le risque de transmission interhumaine, y compris en Europe. Par la suite, plusieurs épidémies ont été rapportées (figure 3), la plus sévère en Angola en 2004-2005, avec une létalité de 88\% (329 décès) $(6,7)$.

\section{La Fièvre de Lassa}

La fièvre de Lassa est causée par un arénavirus, le virus Lassa. On distingue au sein des arénavirus 2 grands complexes selon leur répartition géographique: i) le complexe dit " de l'ancien monde " ou complexe LCML/Lassa (LCML = Chorioméningite lymphocytaire); ii) le complexe américain ou "complexe Tacaribe » regroupant des virus responsables de fièvres virales hémorragiques sur le continent américain : virus Junin (Argentine), Machupo (Bolivie) et Guanarita pour le Vénézuela. II s'agit de virus enveloppés, transmis via les excrétas de rongeurs largement infectés (taux d'infection jusqu'à $80 \%$ ), directement à la population humaine en contact étroit avec ses animaux au sein des villages. Des transmissions nosocomiales sont possibles par contacts avec les fluides biologiques voire par aérosols.

Les premiers cas documentés de fièvre de Lassa remontent à l'année 1969, avec le décès de 2 infirmières missionnaires dans la ville de Lassa, au Nigéria. La fièvre de Lassa est une maladie fréquente, puisqu'on estime que 100000 à 300000 cas surviendraient chaque année en Afrique de l'Ouest (12). C'est également la fièvre hémorragique virale la plus souvent importée en Europe et aux Etats-Unis (au moins 29 cas documentés à ce jour, dont 1/3 sont décédés), la seule pour laquelle une transmission respiratoire semble plausible, et celle pour laquelle on dispose d'un antiviral actif : la 
ribavirine. Le réservoir est un rongeur péri-domestique (Mastomys natalensis), et les zones endémiques sont, d'une part la triade Guinée-Sierra Leone-Libéria (région de la Mano river), et d'autre part le Nigéria (figure 4). Entre ces 2 zones, notamment au sud du Mali et du Burkina Faso, ainsi qu'en Côte d'Ivoire, au Ghana, au Bénin et au Togo, des cas sporadiques sont rapportés, et le virus a pu être isolé chez les rongeurs. Des données récentes suggèrent que le virus de la fièvre de Lassa serait présent dans une zone nettement plus étendue, mais resterait méconnu du fait de la forte proportion de cas asymptomatiques, et de l'absence de moyens affectés à la surveillance de ce virus (13). Du fait de sa large répartition en Afrique de l'Ouest où elle est endémique, la fièvre de Lassa constitue un diagnostic différentiel à la maladie à virus Ebola. Cependant, si la fièvre de Lassa peut indiscutablement se présenter comme une fièvre hémorragique virale sévère, avec un tableau biphasique très proche des fièvres Ebola ou Marburg, les formes asymptomatiques ou pauci-symptomatiques prédominent nettement, et la létalité globale est $<2 \%$.

\section{La Fièvre hémorragique Crimée-Congo}

La virus Crimée-Congo est un nairovirus largement répandu et transmis par des tiques qui constituent un véritable réservoir de ce virus compte-tenu de sa transmission trans-ovarienne chez les tiques. L'homme n'est qu'un hôte accidentel dans ce cycle et est le seul mammifère à développer la maladie. La contamination peut se faire directement au décours de la piqûre par une tique ou indirectement par contact avec le sang ou les viscères des animaux infectés. Selon le mode de contamination, l'incubation varie de 2-5 jours (inoculation du virus par la tique), à 5-14 jours (transmission par contact avec liquides biologiques ou viscères contaminés).

La fièvre hémorragique Crimée-Congo est la plus anciennement décrite et la plus répandue (14, 15). Décrite initialement en Crimée (ex-URSS) en 1944, puis au Congo (ex-Zaïre) en 1956, elle est actuellement à l'état d'endozootie et d'endémie dans de nombreux pays d'Afrique, d'Asie Centrale, de la péninsule Arabique, du moyen Orient et de l'Europe du Sud, l'Europe centrale et l'Europe de l'Est (figure 5). II s'agit d'une arbovirose transmise par des tiques, avec un large spectre, aussi bien en termes de réservoir (de nombreux vertébrés domestiques et sauvages, notamment les ruminants), que de vecteurs (principalement les tiques du genre Hyalomma, mais également Dermacentor et 
Rhipicephalus). En outre, la plupart des vertébrés infectés sont asymptomatiques, ce qui fait que les épizooties passent inaperçues. II s'agit d'une maladie professionnelle chez les éleveurs, les vétérinaires, les travailleurs d'abattoir, mais de nombreuses épidémies nosocomiales ont été rapportées dans des structures de soins où les mesures d'hygiène de base n'avaient pas été respectées $(2,16)$. Comme Ebola et Marburg, la fièvre hémorragique Crimée-Congo ne se transmet pas par voie respiratoire, mais par contact direct avec des liquides biologiques contaminés par du sang en période virémique. La ribavirine ne semble pas efficace $(17,18)$, et le traitement est principalement symptomatique (19), avec une létalité moyenne estimée à 30-50\%, très variable selon les séries (2$80 \%)(14)$.

\section{La Fièvre de la vallée du Rift}

Le virus de la vallée du Rift appartient à la famille de bunyaviridae (virus à ARN), genre phlébovirus. Les premiers cas de fièvre de la vallée du Rift ont été décrits chez des ruminants en 1934 au Kenya. La première épidémie documentée chez l'Homme remonte à 1951, en Afrique du Sud, et a été rattachée à la manipulation de carcasses d'ovins et de bovins (20), principal facteur de risque identifié lors des épidémies chez l'homme (21). Par la suite, de nombreuses épidémies ont été rapportées, souvent à la suite de périodes de pluie intenses, en rapport avec la prolifération des vecteurs, principalement les moustiques Aedes sp., qui agissent comme catalyseur de ces épidémies en accélérant la transmission entre les vertébrés, et des vertébrés à l'homme. Contrairement à la fièvre Crimée-Congo, la fièvre de la vallée du Rift est responsable de maladies sévères chez les ruminants et d'avortements. La surveillance des précipitations permet de prédire le risque d'épidémie de fièvre de la vallée du Rift dans les territoires d'endozootie, avec une bonne précision. Une des épidémies les plus spectaculaires a été rapportée en Egypte en 1977 avec une estimation de 20 à 40000 cas symptomatiques, dont 600 décès. La première épidémie extra-Africaine, survenue sur la péninsule Arabique en 2000 (figure 6), a marqué un tournant et fait craindre une poursuite de l'extension de ce fléau (22). Les formes hémorragiques ne toucheraient que 1 à $3 \%$ des sujets infectés (23). Les formes graves sont d'emblée fulminantes avec une virémie intense et une nécrose hépatique. Ce virus présente également un 
neurotropisme pouvant donner des formes purement neurologiques. Les lésions ophtalmologiques sont une des particularités de cette arbovirose, notamment les rétinites, avec risque de cécité définitive.

\section{Diagnostic biologique des fièvres hémorragiques virales}

A l'exception du virus de la fièvre de la Vallée du Rift, ces pathogènes responsables de fièvres hémorragiques virales entrent dans la catégorie des agents biologiques de classe IV (arrêté modifié du 18/07/1994 fixant la liste des agents biologiques pathogènes pour l'homme), correspondant au risque maximal pour l'homme en termes de dangerosité et de risque de propagation. Cela implique une manipulation au sein de laboratoires de confinement de niveau de sécurité biologique (NSB) 4 (figure 7) selon l'arrêté du 16 juillet 2007) et un transport de ces échantillons en triple emballage (figure 8) par un transporteur agréé selon la réglementation des Matières infectieuses de catégorie A (classe de danger 6-2, UN 2814 « Matières infectieuses pour l'homme »).

Le diagnostic de ces fièvres hémorragiques virales est réalisé par des centres nationaux ou internationaux de référence (CNR) bénéficiant d'une grande expertise dans le domaine. En France, le CNR des fièvres hémorragiques virales se situe à Lyon et dispose d'un laboratoire de type NSB4, le seul existant sur le territoire français (http://www.pasteur.fr/fr/recherche/virologie/unites-etgroupes/biologie-infections-virales-emergentes). Au sein de ces structures spécialisées le diagnostic virologique fait appel à plusieurs types de techniques :

\section{Techniques dites directes:}

- Isolement du virus sur cultures cellulaires (Cellules Vero-Vero 6) avec mise en évidence d'un effet cytopathogène après culture.

- Microscopie électronique (exemple : aspect caractéristique des filovirus, des arénavirus, etc.)

- Mise en évidence d'antigènes viraux spécifiques à l'aide de tests Elisa (possible si grande quantité de virus dans le sang et les tissus)

- RT-PCR en temps réel ciblant des zones spécifiques du génome viral. Cependant en cas de mutation importante sur le génome (variabilité des virus à ARN), la PCR peut être mise en défaut, d'où l'importance d'une surveillance des souches épidémiques par les Centres de Référence (veille épidémiologique). 
2. Techniques dites indirectes, mesurant la réponse en anticorps de type $\lg G$ et $\lg M$. : techniques Elisa (IgG direct, IgM par capture).

En pratique le diagnostic par RT-PCR en temps réel est, en 2015, le test diagnostique le plus sensible, tout en restant spécifique et en permettant un résultat dans les 4 heures suivant la prise en charge de l'échantillon. Ces techniques peuvent être appliquées sur sang prélevé sur tube EDTA, voire sur des urines ou des biopsies tissulaires. La détection d'antigènes viraux par technique Elisa ou par des tests rapides peut être utile sur le terrain, en zones peu équipées ou largement dépassées par l'afflux de patients. Les antigènes viraux et l'ARN viral apparaissent rapidement après le début des

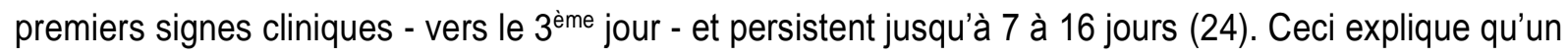
résultat de PCR négatif ne peut exclure le diagnostic si il a été effectué sur un prélèvement obtenu dans les 48 heures après le début des signes cliniques : il devra être contrôlé sur un $2^{\text {ème }}$ prélèvement.

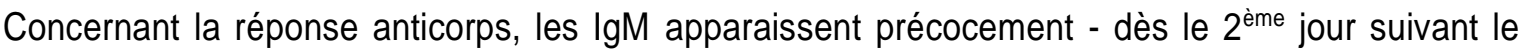
début des signes - et persistent entre 30 et 168 jours. Les lgG se positivent entre le $6^{\text {ème }}$ et le $18^{\text {ème jour }}$ et persistent plusieurs années (24). II est important d'obtenir un $2^{\text {ème }}$ prélèvement sérologique à distance afin de suivre la cinétique des anticorps (séroconversion en $\lg G$, ascension des titres d'anticorps). L'apparition des IgG est souvent trop tardive pour avoir un intérêt diagnostique immédiat, mais ce dosage s'avère intéressant pour des études de séroprévalence dans la population. Ainsi, lors de l'épidémie d'Ebola en Afrique de l'Ouest en 2014-2015, l'analyse des marqueurs sérologiques a permis de mettre en évidence un profil sérologique chez les patients décédés différents des survivants. Ces derniers développaient une immunité humorale très rapidement, constituant ainsi un marqueur pronostique (25).

L'épidémie récente d'Ebola a soulevé de nombreuses interrogations et amené nos autorités sanitaires et sociétés savantes à émettre des recommandations en matière de prise en charge des échantillons biologiques. Les plus officielles émanent du Haut Conseil de la Santé Publique (HCSP) et sont libres d'accès sur le site (http://www.hcsp.fr/explore.cgi/avisrapportsdomaine?clefr=414). En France, en 2014 seul le laboratoire NSB4 de Lyon était habilité à réaliser le diagnostic virologique de la 
MVE. Afin de ne pas retarder la prise en charge du patient, les 12 Etablissements de Santé de Référence (ESR), répartis sur le territoire métropolitain et Outre-Mer, se devaient de pouvoir réaliser

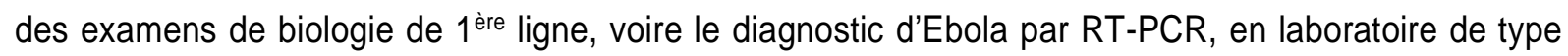
NSB 3, sous poste de sécurité microbiologique de niveau III ou PSM III (figure 9), selon l'arrêté du 6 août 2014 modifiant l'arrêté de 2007. Ce transfert de technologie s'est fait en lien étroit avec le CNR selon un cahier des charges bien établi.

\section{Conclusions}

Les fièvres hémorragiques virales forment un groupe de maladies virales hétérogènes, potentiellement graves, avec un potentiel épidémique illustré de manière dramatique par l'épidémie Ebola qui a frappé l'Afrique de l'Ouest en 2014-2015. Si le tableau clinique des formes hémorragiques est relativement stéréotypé, avec une évolution biphasique (syndrome pseudo-grippal initial pouvant évoluer, ou pas, vers un syndrome hémorragique), la biologie de ces virus et leur épidémiologie sont nettement plus variables, et nécessitent des mesures adaptées en termes de surveillance et de prévention. L'importance d'un diagnostic rapide, disponible sur le terrain, abordable et facile d'utilisation réside plus dans la nécessité d'interrompre les chaines de transmission avant qu'il ne soit trop tard, que dans les possibilités thérapeutiques, qui restent très limitées en 2015. 
Tableau I : Caractéristiques des fièvres hémorragiques virales

\begin{tabular}{|c|c|c|c|c|c|c|c|}
\hline Famille & \multicolumn{2}{|c|}{ Filoviridae } & \multicolumn{3}{|c|}{ Arenaviridae } & \multicolumn{2}{|c|}{ Bunyaviridae } \\
\hline Genre & $\begin{array}{l}\text { Filovirus } \\
\text { (Figure 1) }\end{array}$ & Filovirus & $\begin{array}{l}\text { Complexe } \\
\text { Tacaribe }\end{array}$ & $\begin{array}{l}\text { Complexe } \\
\text { Tacaribe }\end{array}$ & $\begin{array}{l}\text { complexe } \\
\text { LCMV/Lassa }\end{array}$ & Nairovirus & Phlébovirus \\
\hline Nom & Ebola & Marburg & Junin & Machupo & Lassa & $\begin{array}{l}\text { Crimée- } \\
\text { Congo }\end{array}$ & Vallée du Rift \\
\hline Géographie & $\begin{array}{l}\text { Afrique } \\
\text { (figure 2) }\end{array}$ & $\begin{array}{l}\text { Afrique } \\
\text { (figure 3) }\end{array}$ & $\begin{array}{l}\text { Amérique } \\
\text { du Sud }\end{array}$ & $\begin{array}{l}\text { Amérique } \\
\text { du Sud }\end{array}$ & $\begin{array}{c}\text { Afrique } \\
\text { de l'Ouest } \\
\text { (figure 4) }\end{array}$ & $\begin{array}{c}\text { Afrique, Asie } \\
\text { centrale, } \\
\text { Europe, } \\
\text { Moyen Orient } \\
\text { (figure 5) }\end{array}$ & $\begin{array}{c}\text { Afrique, Yémen, } \\
\text { Arabie Saoudite } \\
\text { (figure 6) }\end{array}$ \\
\hline Réservoir & $\begin{array}{c}\text { Chauves- } \\
\text { souris, singes }\end{array}$ & $\begin{array}{c}\text { Chauves-souris, } \\
\text { singes }\end{array}$ & $\begin{array}{l}\text { Rongeurs } \\
\text { (Mastomys } \\
\text { natalensis) }\end{array}$ & Rongeurs & Rongeurs & $\begin{array}{l}\text { Vertébrés } \\
\text { domestiques } \\
\text { et sauvages }\end{array}$ & Ruminants \\
\hline Vecteur & Non & Non & Non & Non & Non & $\begin{array}{c}\text { Tiques } \\
\text { (Hyalomma) }\end{array}$ & $\begin{array}{l}\text { Moustiques } \\
\text { (Aedes sp.) }\end{array}$ \\
\hline $\begin{array}{l}\text { Incubation } \\
\text { (jours) }\end{array}$ & $2-21$ & $2-21$ & $7-14$ & $9-15$ & $5-21$ & 3-14 & $2-6$ \\
\hline Début & Brutal & Brutal & Progressif & Progressif & Progressif & Progressif & Brutal \\
\hline Particularités & $\begin{array}{l}\text { Létalité } \\
(40-90 \%) \\
\text { Bouffées } \\
\text { épidémiques }\end{array}$ & $\begin{array}{l}\text { Létalité } \\
(30-90 \%) \\
\text { Bouffées } \\
\text { épidémiques }\end{array}$ & Encéphalites & Encéphalites & $\begin{array}{l}\text { Rareté de la } \\
\text { thrombopénie } \\
\text { Létalité faible (1- } \\
\text { 2\%) }\end{array}$ & $\begin{array}{l}\text { Létalité } 30 \text { - } \\
\qquad 50 \%\end{array}$ & $\begin{array}{c}\text { Létalité }<10 \% \\
\text { Atteintes } \\
\text { occulaires }\end{array}$ \\
\hline $\begin{array}{l}\text { Traitement } \\
\text { antiviral }\end{array}$ & $\begin{array}{c}\text { Aucun } \\
\text { d'efficacité } \\
\text { démontrée }\end{array}$ & Aucun & Ribavirine & $?$ & Ribavirine & $\begin{array}{l}\text { Ribavirine } \\
\text { sans doute } \\
\text { inefficace }\end{array}$ & Ribavirine \\
\hline
\end{tabular}


Figure 1. Virus Ebola en microscopie électronique

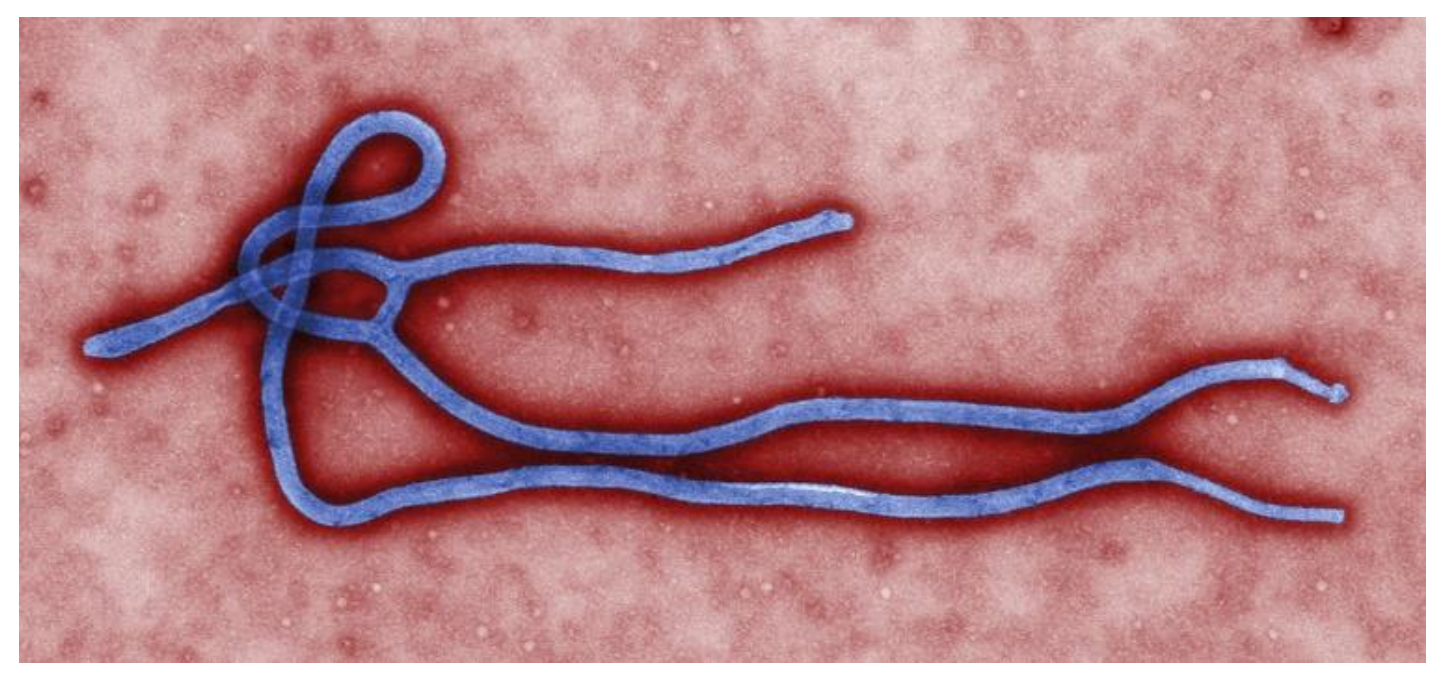

Source : REUTERS/Cynthia Goldsmith/CDC/Handout (http://www.cdc.gov) 
Figure 2. Les épidémies 'Ebola' de 1976 à 2015 (d'après Centers for Disease Control and prevention, http://www.cdc.gov)

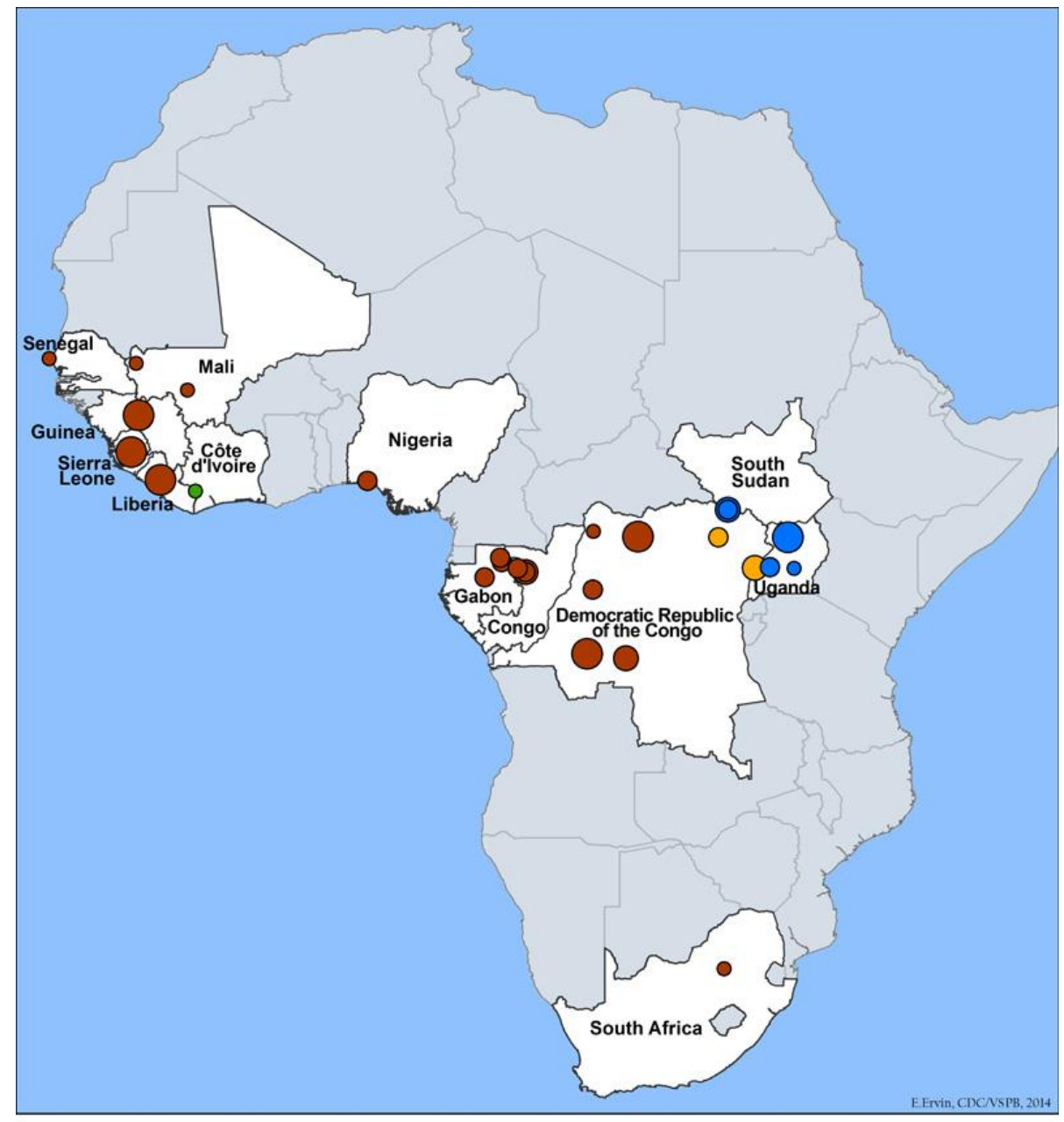

EBOLAVIRUS OUTBREAKS BY SPECIES AND SIZE, 1976 - 2014

Species

- Zaire ebolavirus

- Sudan ebolavirus

- Tai Forest ebolavirus

Bundibugyo ebolavirus
Number of Cases

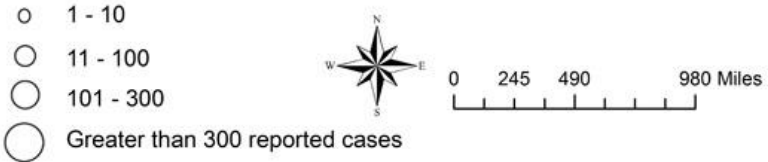


Figure 3. Les épidémies 'Marburg' de 1967 à 2012 (d'après Centers for Disease Control and prevention, http://www.cdc.gov)

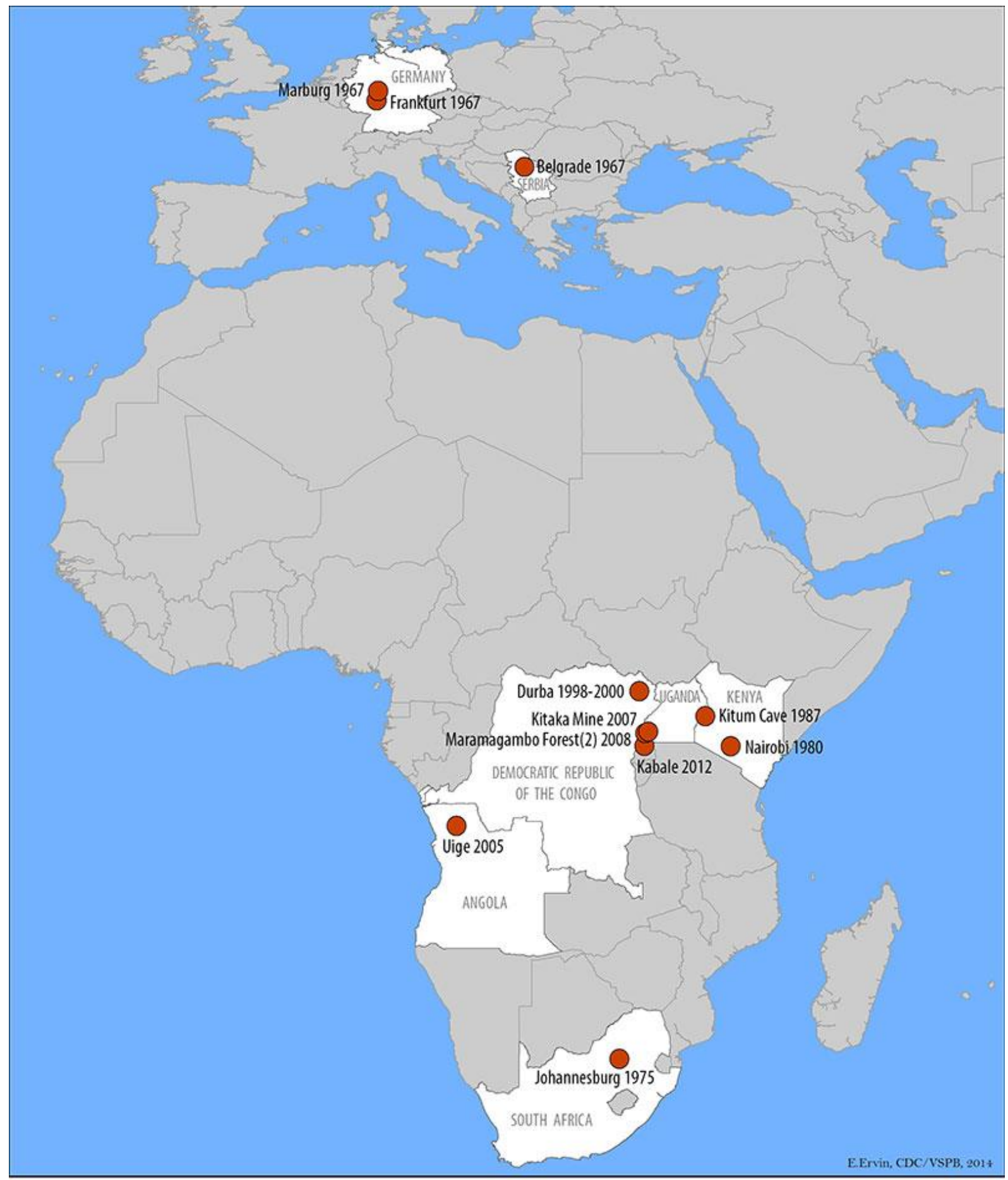

PREVIOUS OUTBREAKS OF MARBURG HEMORRHAGIC FEVER

Outbreak location and year

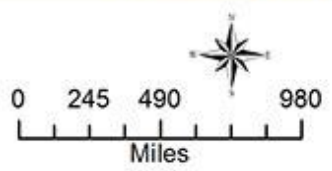


Figure 4. Répartition géographique de la fièvre de Lassa (d'après Centers for Disease Control and prevention, http://www.cdc.gov)

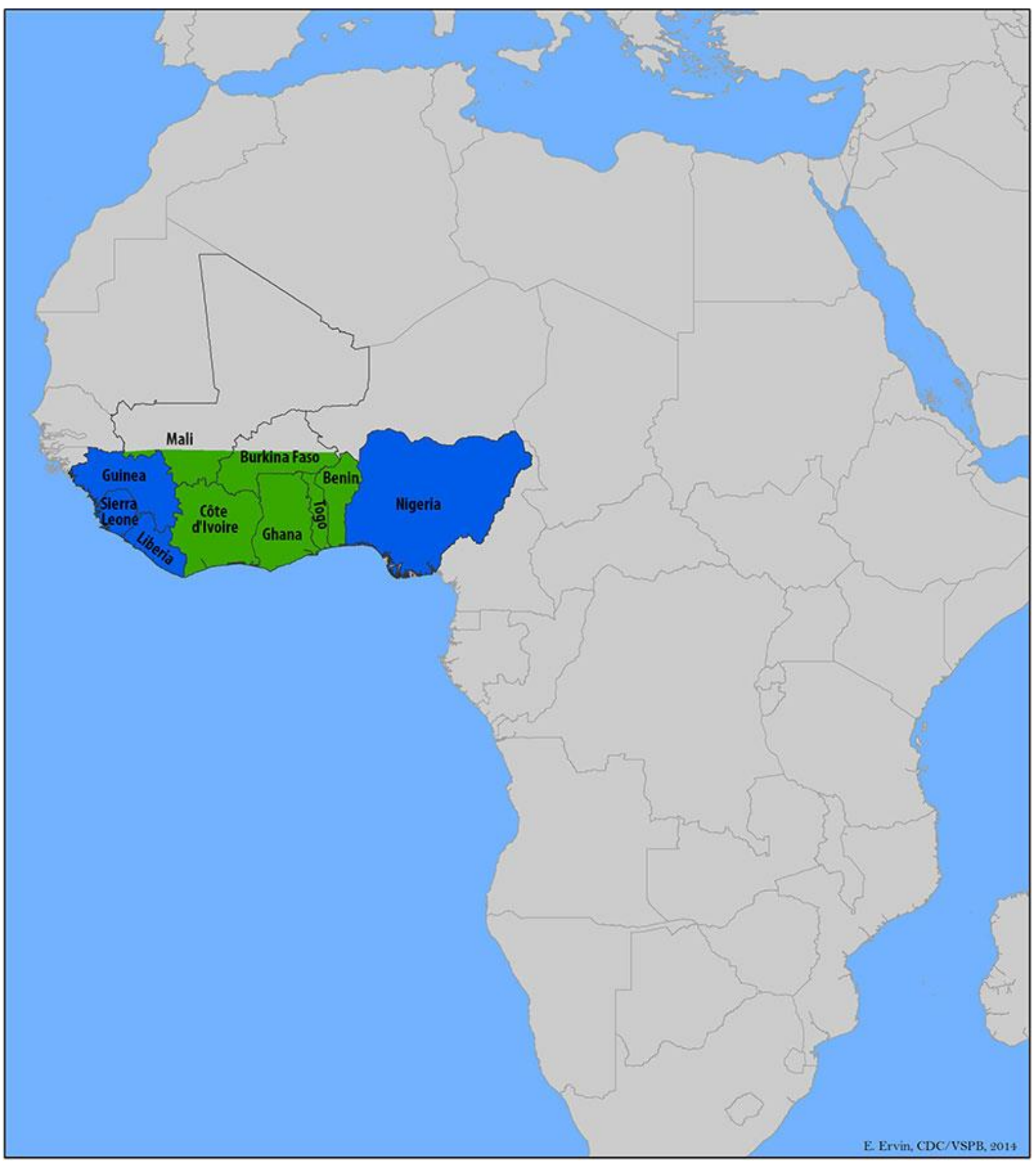

\section{LASSA FEVER DISTRIBUTION MAP}

Countries reporting endemic disease and substantial outbreaks of Lassa Fever

Countries reporting few cases, periodic isolation of virus, or serologic evidence of Lassa virus infection

Lassa Fever status unknown

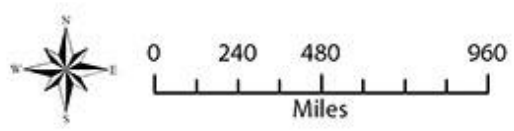


Figure 5. Répartition géographique de la fièvre hémorragique Crimée-Congo (d'après Centers for Disease Control and prevention, http://www.cdc.gov)

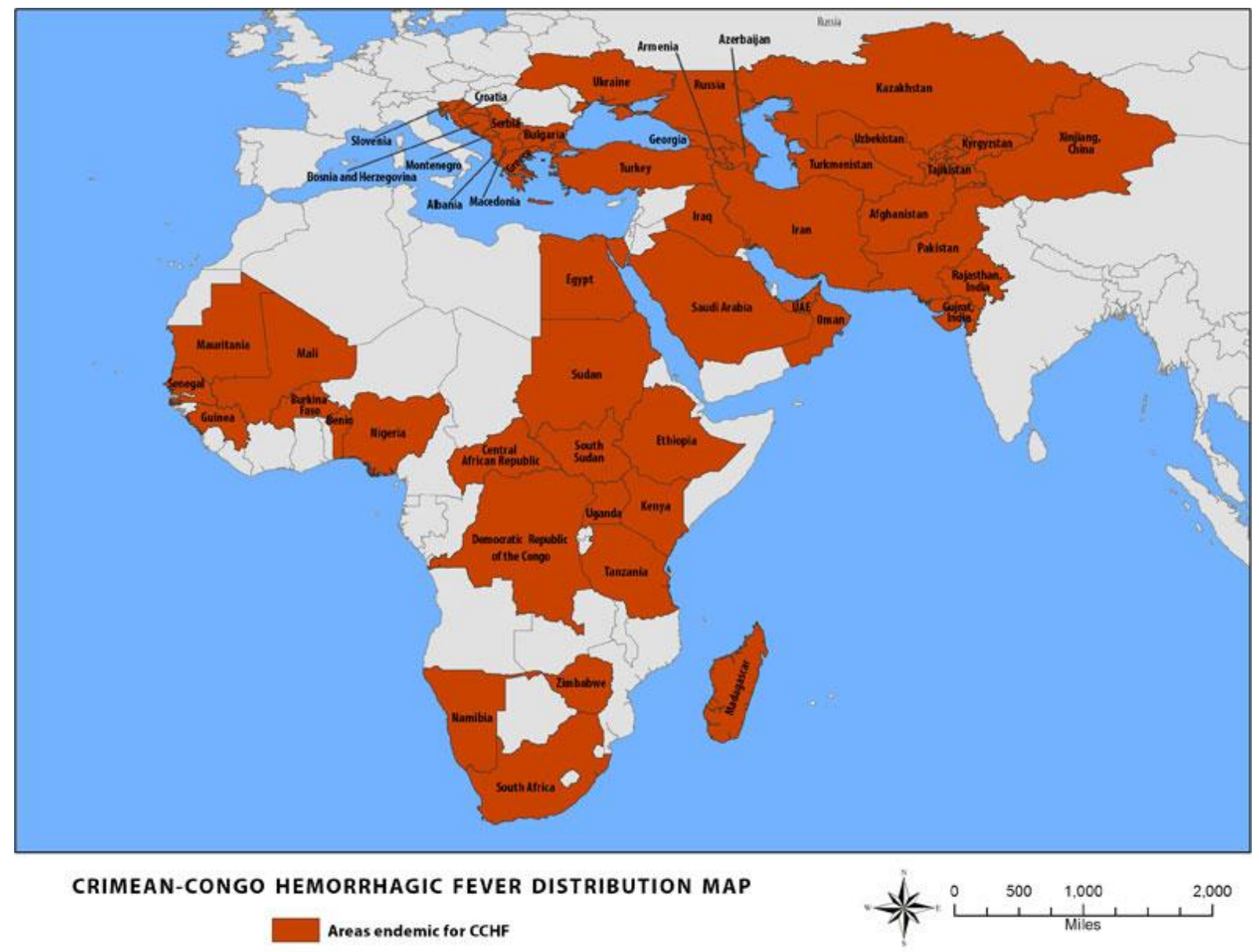


Figure 6. Répartition géographique de la fièvre de la Vallée du Rift (d'après Centers for Disease Control and prevention, http://www.cdc.gov)

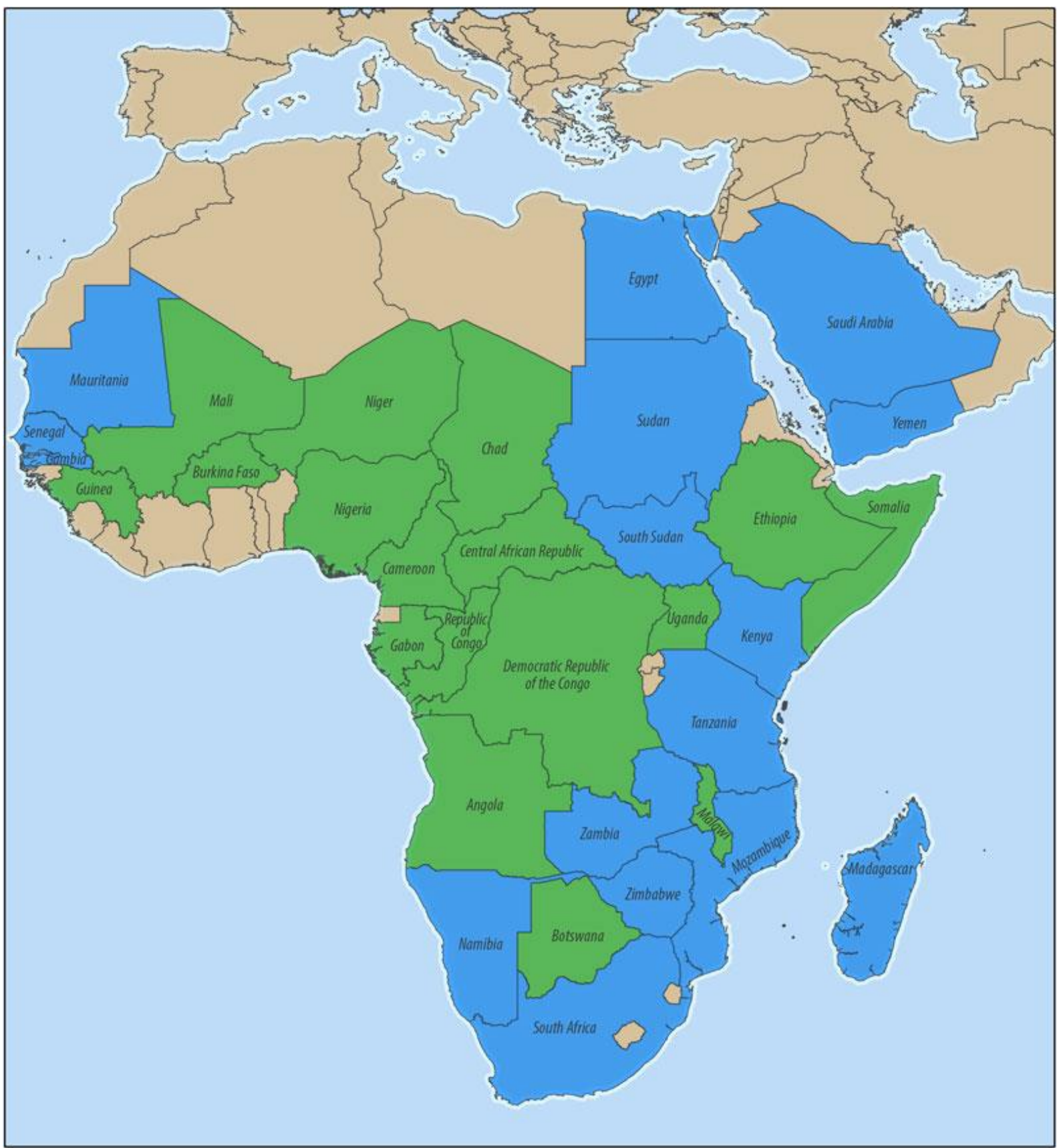

\section{RIFT VALLEY FEVER DISTRIBUTION MAP}

Countries reporting endemic disease and substantial outbreaks of RVF

Countries reporting few cases, periodic isolation of virus, or serologic evidence of RVF infection

RVF status unknown

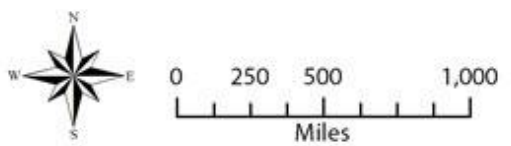


Figure 7. Laboratoire de confinement de niveau de sécurité biologique (NSB) 4

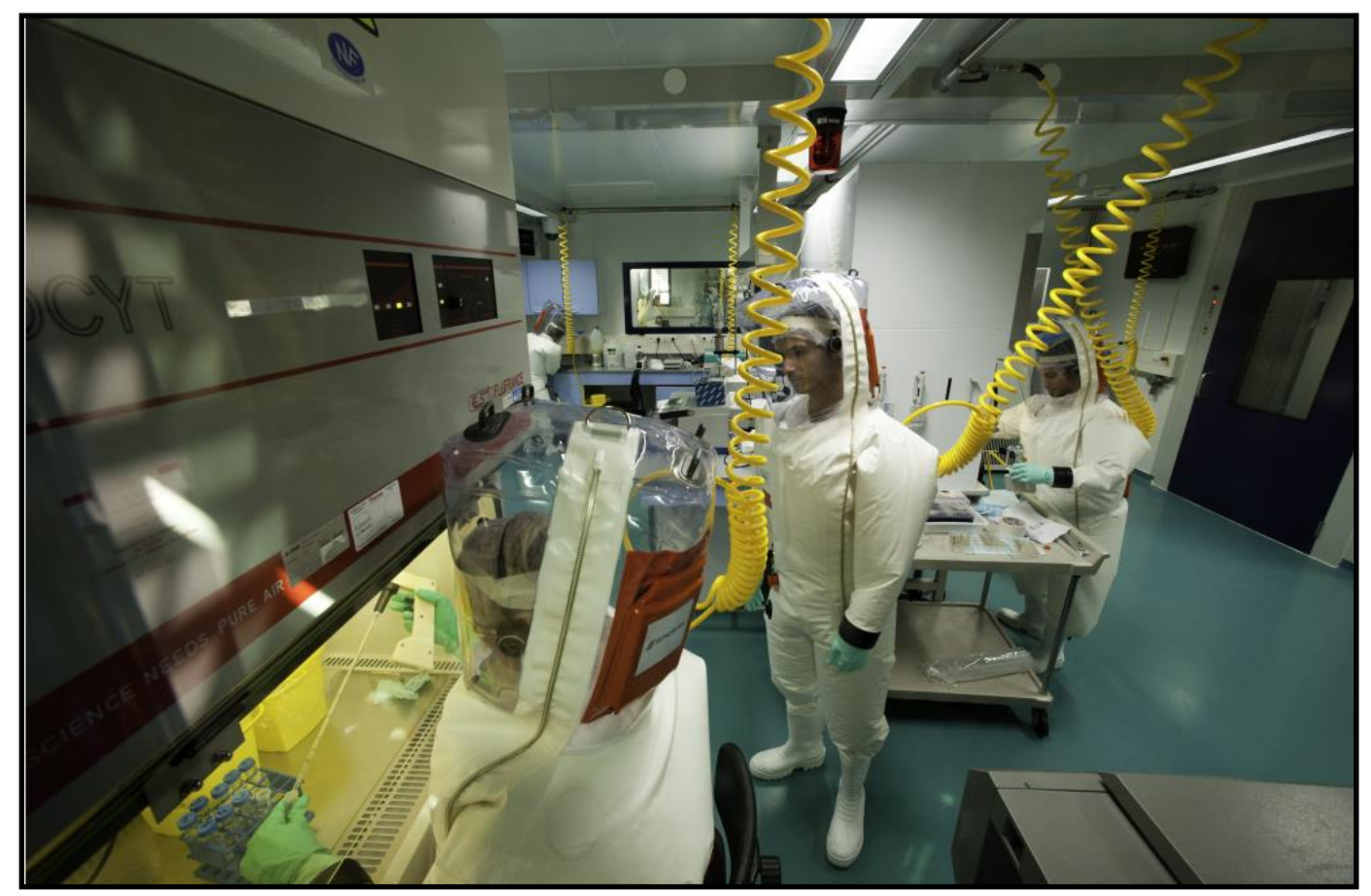


Figure 8. Triple emballage UN2814

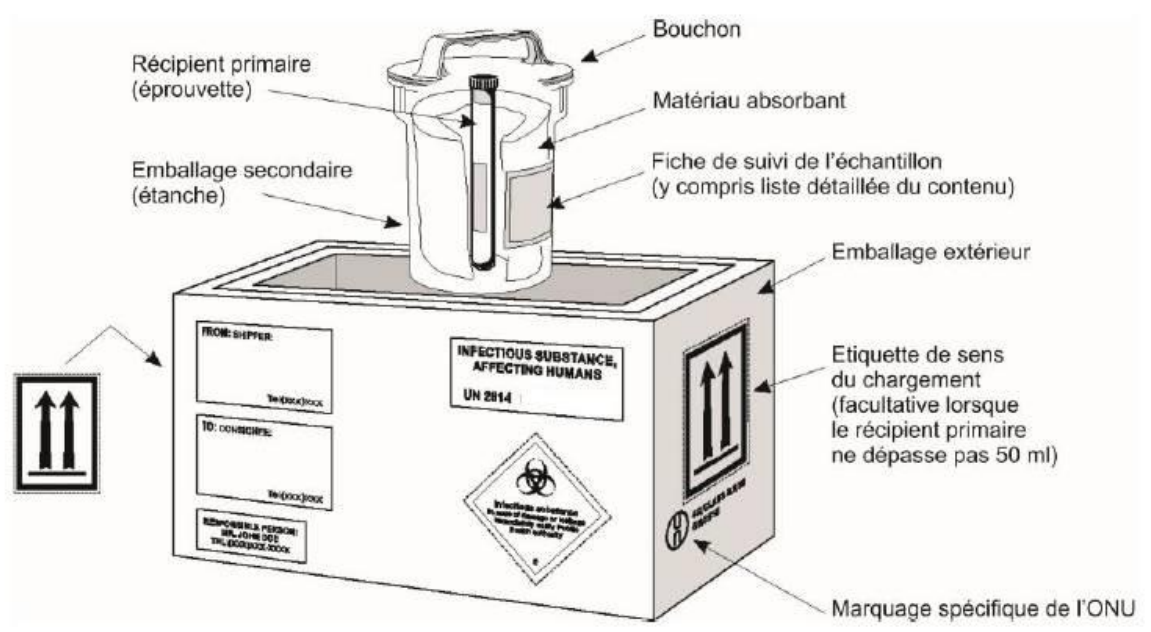


Figure 9. Poste de Sécurité Microbiologique (PSM) de type III

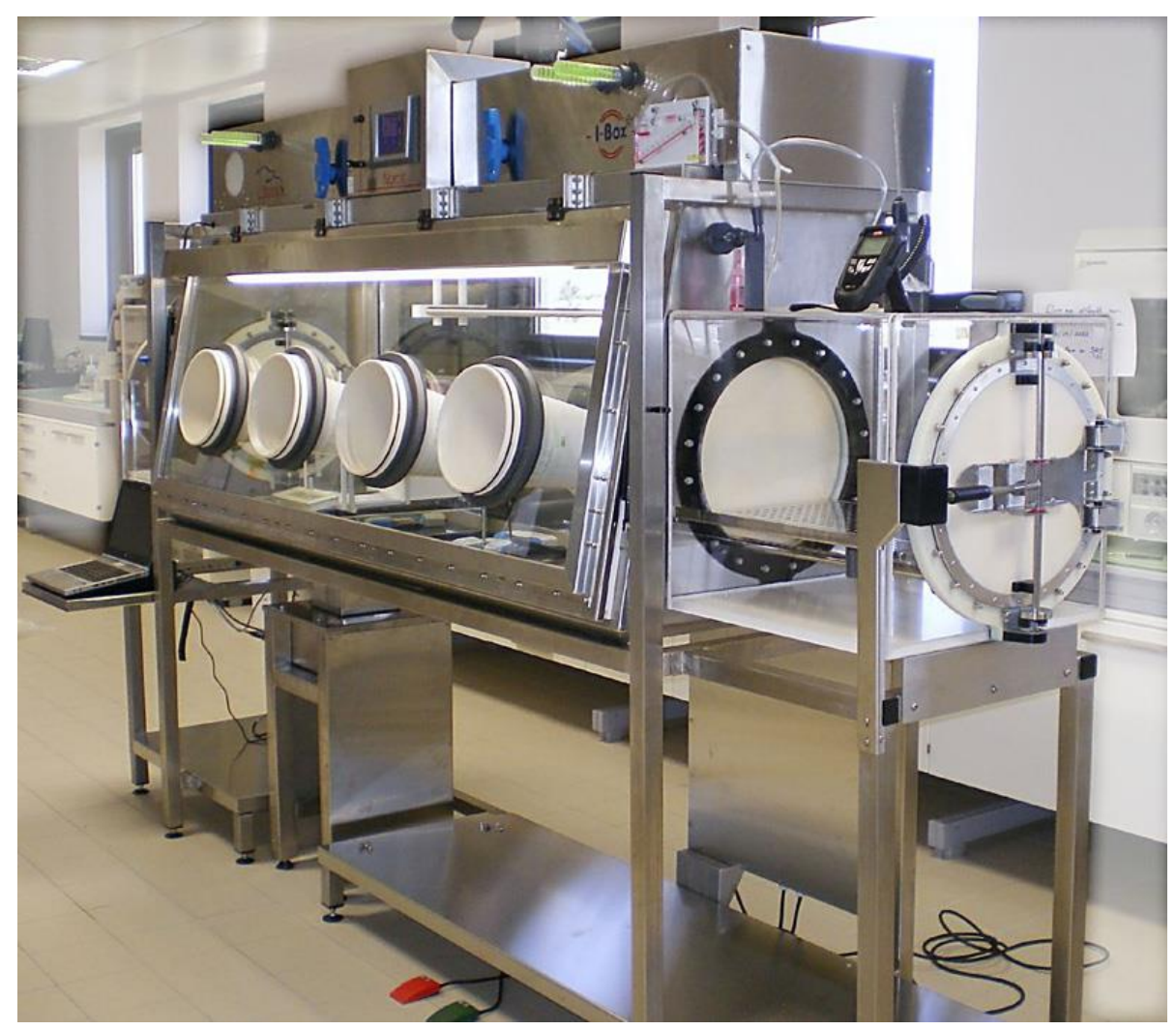


A. Parmi les fièvres hémorragiques virales suivantes, laquelle(lesquelles) est(sont) une(des) arbovirose(s) ?

1. Fièvre de Lassa

2. Fièvre hémorragique Crimée-Congo

3. Fièvre de Marburg

4. Fièvre de la vallée du Rift

5. Maladie virale Ebola

B. Parmi les fièvres hémorragiques virales suivantes, laquelle est actuellement présente en Afrique, Asie et Europe?

1. Fièvre de Lassa

2. Fièvre hémorragique Crimée-Congo

3. Fièvre de Marburg

4. Fièvre de la vallée du Rift

5. Maladie virale Ebola

C. Parmi les fièvres hémorragiques virales suivantes, laquelle est transmissible par voie respiratoire?

1. Fièvre de Lassa

2. Fièvre hémorragique Crimée-Congo

3. Fièvre de Marburg

4. Fièvre de la vallée du Rift

5. Maladie virale Ebola 
D. Parmi les fièvres hémorragiques virales suivantes, citez les 3 qui sont associées aux taux de létalité* les plus élevés?

1. Fièvre de Lassa

2. Fièvre hémorragique Crimée-Congo

3. Fièvre de Marburg

4. Fièvre de la vallée du Rift

5. Maladie virale Ebola

* risque de mourir lorsque l'on est infecté

E. Parmi les fièvres hémorragiques virales suivantes, laquelle est probablement améliorée par un traitement par ribavirine?

1. Fièvre de Lassa

2. Fièvre hémorragique Crimée-Congo

3. Fièvre de Marburg

4. Fièvre de la vallée du Rift

5. Maladie virale Ebola

Résultats:
A: 2,4
B: 2
C: 1
D: $2,3,5$
E: 1 


\section{Références}

1. Jaureguiberry S, Tattevin P, Tarantola A, Legay F, Tall A, Nabeth P, et al. Imported Crimean-Congo hemorrhagic Fever. Journal of clinical microbiology. 2005;43(9):4905-7.

2. Tarantola A, Nabeth P, Tattevin P, Michelet C, Zeller H. Lookback exercise with imported Crimean-Congo hemorrhagic fever, Senegal and France. Emerg Infect Dis. 2006;12(9):1424-6.

3. Agua-Agum J, Ariyarajah A, Aylward B, Blake IM, Brennan R, Cori A, et al. West African Ebola epidemic after one year--slowing but not yet under control. The New England journal of medicine. 2015;372(6):584-7.

4. Meyers L, Frawley T, Goss S, Kang C. Ebola virus outbreak 2014: clinical review for emergency physicians. Annals of emergency medicine. 2015;65(1):101-8.

5. Feldmann H, Geisbert TW. Ebola haemorrhagic fever. Lancet (London, England). 2011;377(9768):849-62.

6. Leroy E, Baize S, Gonzalez JP. [Ebola and Marburg hemorrhagic fever viruses: update on filoviruses]. Medecine tropicale : revue du Corps de sante colonial. 2011;71(2):111-21.

7. Leroy EM, Gonzalez JP, Baize S. Ebola and Marburg haemorrhagic fever viruses: major scientific advances, but a relatively minor public health threat for Africa. Clinical microbiology and infection : the official publication of the European Society of Clinical Microbiology and Infectious Diseases. 2011;17(7):964-76.

8. Baize S, Pannetier D, Oestereich L, Rieger T, Koivogui L, Magassouba N, et al. Emergence of Zaire Ebola virus disease in Guinea. The New England journal of medicine. 2014;371(15):1418-25.

9. Tattevin P, Durante-Mangoni E, Massaquoi M. Does this patient have Ebola virus disease? Intensive care medicine. 2014;40(11):1738-41. 
10. Yazdanpanah Y, Arribas JR, Malvy D. Treatment of Ebola virus disease. Intensive care medicine. 2015;41(1):115-7.

11. Rougeron V, Feldmann H, Grard G, Becker S, Leroy EM. Ebola and Marburg haemorrhagic fever. Journal of clinical virology : the official publication of the Pan American Society for Clinical Virology. 2015;64:111-9.

12. Leparc-Goffart I, Emonet SF. [An update on Lassa virus]. Medecine tropicale : revue du Corps de sante colonial. 2011;71(6):541-5.

13. Sogoba N, Feldmann H, Safronetz D. Lassa fever in West Africa: evidence for an expanded region of endemicity. Zoonoses and public health. 2012;59 Suppl 2:43-7.

14. Oncu S. Crimean-Congo hemorrhagic fever: an overview. Virologica Sinica. 2013;28(4):193-201.

15. Ergonul O. Crimean-Congo hemorrhagic fever virus: new outbreaks, new discoveries. Current opinion in virology. 2012;2(2):215-20.

16. Ftika L, Maltezou HC. Viral haemorrhagic fevers in healthcare settings. The Journal of hospital infection. 2013;83(3):185-92.

17. Ascioglu S, Leblebicioglu H, Vahaboglu H, Chan KA. Ribavirin for patients with Crimean-Congo haemorrhagic fever: a systematic review and meta-analysis. The Journal of antimicrobial chemotherapy. 2011;66(6):1215-22.

18. Ergonul O, Celikbas A, Dokuzoguz B, Eren S, Baykam N, Esener H. Characteristics of patients with Crimean-Congo hemorrhagic fever in a recent outbreak in Turkey and impact of oral ribavirin therapy. Clin Infect Dis. 2004;39(2):284-7.

19. Flusin O, Iseni F, Rodrigues R, Paranhos-Baccala G, Crance JM, Marianneau P, et al. [Crimean-Congo hemorrhagic fever: basics for general practitioners]. Medecine tropicale : revue du Corps de sante colonial. 2010;70(5-6):429-38. 
20. Davies FG. The historical and recent impact of Rift Valley fever in Africa. The American journal of tropical medicine and hygiene. 2010;83(2 Suppl):73-4.

21. Mohamed M, Mosha F, Mghamba J, Zaki SR, Shieh WJ, Paweska J, et al. Epidemiologic and clinical aspects of a Rift Valley fever outbreak in humans in Tanzania, 2007. The American journal of tropical medicine and hygiene. 2010;83(2 Suppl):22-7.

22. Chevalier V, Pepin M, Plee L, Lancelot R. Rift Valley fever--a threat for Europe? Euro surveillance : bulletin Europeen sur les maladies transmissibles = European communicable disease bulletin. 2010;15(10):19506.

23. Pepin M. [Rift Valley fever]. Med Mal Infect. 2011;41(6):322-9.

24. Wang YP, Zhang XE, Wei HP. Laboratory detection and diagnosis of filoviruses. Virologica Sinica. 2011;26(2):73-80.

25. Goeijenbier M, van Kampen JJ, Reusken CB, Koopmans MP, van Gorp EC. Ebola virus disease: a review on epidemiology, symptoms, treatment and pathogenesis. The Netherlands journal of medicine. 2014;72(9):442-8. 